\title{
A THEOREM ON MAPPINGS INTO RIEMANN SURFACES OF INFINITE GENUS $\left({ }^{1}\right)$ \\ BY \\ LEO SARIO
}

1. Consider complex analytic mappings $f$ of a noncompact Riemann surface $R$ into a compact or noncompact Riemann surface $S$ of finite or infinite genus. If the integrated Euler characteristic $E(\Omega)$ of an exhausting subregion $\Omega \subset R$ does not grow more rapidly than the characteristic function $C(\Omega)$ of $f$, then the defect sum is finite [2]. In the general case there can be infinitely many Picard points and nothing is known about the set of defect points.

We shall show that the defect points under every $f$ are so scarce that

$$
\int_{S} B(k, a) d \omega(a)=O(k) .
$$

Here $k$ is the value on $\partial \Omega$ of the capacity function of $\Omega, B$ stands for the proximity function to $a \in S$ on $\partial \Omega$, and $d \omega(a)$ is the area element in a conformal metric with finite total area. The significance of this result will be discussed in Nos. 12,16 below.

The proof, based on principal functions, will be decomposed into Lemmas 1-9.

\section{§1. Construction OF THE PROXIMITY FUnCtion.}

2. Let $H$ be the class of harmonic functions and let $E$ be a compact set on a Riemann surface $F$. There exists [2] a constant $q \in(0,1)$ such that $\max _{E}|u| \leqq q \sup _{F}|u|$ for all $u \in H$ on $F$ with nonconstant sgn $u \mid E$.

3. Let $W$ be an arbitrary Riemann surface and $W_{0} \subset W$ a regular region with border $\alpha_{0}$. Take a noncompact bordered region $W_{1} \subset W$ with compact border $\alpha_{1} \subset W_{0}$ and with compact complement $W-W_{1} \subset W_{0}$. On $\bar{W}_{1}$ let $\sigma \in H$ with $\sigma \mid \alpha_{1}=0, \int_{\alpha_{1}} d \sigma^{*}=0$, and set $m=\min _{\alpha_{0}} \sigma, M=\max _{\alpha_{0}} \sigma$. Let $q$ be the constant of No. 2 for $E=\alpha_{1}, F=W_{0}$, and write $Q=(1-q)^{-1}$. Denote by $L$ a normal operator, i.e., a linear operator associating with each $\phi \in H$ on $\alpha_{1}$ a unique harmonic functon $L \phi$ on $W_{1}$ with $L \phi \mid \alpha_{1}=\phi$ and such that $\min \phi \leqq L \phi \leqq \max \phi$ and $\int_{\alpha_{1}} d^{*}(L \phi)=0$. The subsequent construction will be based on the following general existence theorem [2], [3]:

There is a function $p \in H$ on $W$, called the principal function, such that

Received by the editors October 22, 1963.

(1) Sponsored by the U.S. Army Research Office(Durham), Grant DA-ARO(D)-31-124-G499, University of California, Los Angeles. 


$$
\begin{aligned}
p \mid W_{1} & =\sigma+L p, \\
Q m & \leqq p-\sigma \leqq Q M, \\
Q m & \leqq p \mid W_{0} \leqq Q M .
\end{aligned}
$$

The function is unique up to an additive constant.

4. To construct proximity functions on an arbitrary Riemann surface $S$ choose an arbitrary but then fixed normal operator, e.g. $L_{1}$ [1]. Take parametric disks $D_{0}, D_{1}$ centered at $\zeta_{0}, \zeta_{1}$, and a boundary neighborhood $D_{\beta}$ of $S$, with closures disjoint by pairs. Let $t_{0}$ be the principal function corresponding to $W_{1}=\left(D_{0}-\zeta_{0}\right) \cup\left(D_{1}-\zeta_{1}\right) \cup D_{\beta}$ and $\sigma\left|\bar{D}_{0}-\zeta_{0}=-2 \log \right| \zeta-\zeta_{0} \mid$, $\sigma\left|\bar{D}_{1}-\zeta_{1}=2 \log \right| \zeta-\zeta_{1}|, \sigma| \bar{D}_{\beta}=0$. Normalize by $t_{0}-\sigma \rightarrow 0$ as $\zeta \rightarrow \zeta_{0}$ and set $s_{0}=\log \left(1+e^{t_{0}}\right)$.

For $a \neq \zeta_{0}, D_{a}$ centered at $a$, let $t(\zeta, a)$ be the principal function that corresponds to $\sigma\left|\bar{D}_{a}-a=-2 \log \right| \zeta-a|, \sigma| \bar{D}_{0}-\zeta_{0}=2 \log \left|\zeta-\zeta_{0}\right|, \sigma \mid \bar{D}_{\beta}=0$. For the normalization we now choose $t-\sigma \rightarrow s_{0}(a)$ as $\zeta \rightarrow \zeta_{0}$. Our proximity function is $s=s_{0}+t$.

\section{§2. SyMmetry OF THE PROXIMITY FUNCTION.}

5. Suppose for a moment that $S$ is a bordered Riemann surface with compact border $\beta$. Let $V$ be the class of harmonic functions $v$ on $S-\zeta_{0}-a$ with the same singularities and normalization as $t$ :

$$
\begin{aligned}
& v \mid \bar{D}_{a}-a=-2 \log r+h, \\
& v \mid \bar{D}_{0}-\zeta_{0}=2 \log r+k,
\end{aligned}
$$

where $h, k \in H$ and $k\left(\zeta_{0}\right)=s_{0}(a)$. Set $c=4 \pi h(a)$. In $V$ single out the functions $v_{0}, v_{1}$ determined by

$$
\frac{\partial v_{0}}{\partial n}\left|\beta=0, \quad v_{1}\right| \beta=\text { const. }
$$

and set for real $\lambda$ :

$$
v_{\lambda}=(1-\lambda) v_{0}+\lambda v_{1} .
$$

The quantities $h, k, c$ corresponding to $v_{\lambda}$ will be denoted by $h_{\lambda}, k_{\lambda}, c_{\lambda}$. For $v, v^{\prime} \in V$ we write $B(v)=\int_{\beta} v d v^{*}, B\left(v, v^{\prime}\right)=\int_{\beta} v d v^{\prime *}$.

LEMMA 1. The function $v_{\lambda}$ minimizes $B(v)-(2 \lambda-1) c$ in $V$ :

$$
B(v)+(2 \lambda-1) c=\lambda^{2} c_{1}-(1-\lambda)^{2} c_{0}+D\left(v-v_{\lambda}\right) .
$$

Proof. The Dirichlet integral of $v-v_{\lambda}$ over $S$ is $D\left(v-v_{\lambda}\right)=B(v)+B\left(v_{\lambda}\right)$ - $B\left(v, v_{\lambda}\right)-B\left(v_{\lambda}, v\right)$. Let $A_{i}(v), i=1,2$, be the integral $\int v d v^{*}$ along $\partial D_{a}, \partial D_{0}$, respectively, and similarly for $A_{i}\left(v, v^{\prime}\right)$. Then 


$$
\begin{aligned}
B\left(v_{\lambda}\right) & =(1-\lambda) \lambda B\left(v_{0}, v_{1}\right) \\
& =(1-\lambda) \lambda\left(B\left(v_{0}, v_{1}\right)-B\left(v_{1}, v_{0}\right)\right) \\
& =(1-\lambda) \lambda \sum_{1}^{2}\left(A_{i}\left(v_{0}, v_{1}\right)-A_{i}\left(v_{1}, v_{0}\right)\right) .
\end{aligned}
$$

Here the first summand is $A_{1}\left(h_{0},-2 \log r\right)-A_{1}\left(h_{1},-2 \log r\right)=c_{1}-c_{0}$. Because of the normalization at $\zeta_{0}$, the second summand vanishes and we have

$$
B\left(v_{\lambda}\right)=(1-\lambda) \lambda\left(c_{1}-c_{0}\right) .
$$

Similarly

$$
\begin{aligned}
& B\left(v, v_{\lambda}\right)=\lambda B\left(v, v_{1}\right)=\lambda\left(c_{1}-c\right) \\
& B\left(v_{\lambda}, v\right)=(1-\lambda) B\left(v_{0}, v\right)=(1-\lambda)\left(c-c_{0}\right) .
\end{aligned}
$$

Equality (5) follows.

6. Now let $S$ be an arbitrary noncompact Riemann surface, and $\Omega$ a regular subregion with border $\beta_{\Omega}$. Let $v_{\lambda \Omega}$ be the function (4) constructed on $\Omega$ as above.

Lemma 2. The directed limit

$$
v_{\lambda}=\lim _{\Omega \rightarrow S} v_{\lambda \Omega}
$$

exists and the convergence is uniform in compact subsets of $S-a-\zeta_{0}$.

Proof. Let $\Omega \subset \Omega^{\prime}$ and indicate by primes the quantities corresponding to $\Omega^{\prime}$ or $\beta_{\Omega^{\prime}}$. We apply (5) to $v=v_{0}^{\prime}, v_{\lambda}=v_{0}$ and obtain

$$
B\left(v_{0}^{\prime}\right)-c_{0}^{\prime}=-c_{0}+D\left(v_{0}^{\prime}-v_{0}\right) \text {. }
$$

For $v=v_{1}^{\prime}, v_{\lambda}=v_{1}$ we have

$$
B\left(v_{1}^{\prime}\right)+c_{1}^{\prime}=c_{1}+D\left(v_{1}^{\prime}-v_{1}\right) .
$$

Similarly, $v=v_{1}, v_{\lambda}=v_{0}$ gives

$$
B\left(v_{1}\right)-c_{1}=-c_{0}+D\left(v_{1}-v_{0}\right) \text {. }
$$

From these equations and from the relations $B\left(v_{i}^{\prime}\right) \leqq B^{\prime}\left(v_{i}^{\prime}\right)=0, B\left(v_{1}\right)=0$, $D \geqq 0$, we infer that $c_{1}$ increases while $c_{0}$ decreases with increasing $\Omega$, and $c_{1} \leqq c_{0}$ for every $\Omega$. A fortiori the limit $c_{i}=\lim c_{i \Omega}$ exists, with obvious meaning of $c_{i \Omega}$. This implies

$$
\lim _{\Omega \rightarrow S} D_{\Omega}\left(v_{i \Omega^{\prime}}-v_{i \Omega}\right)=0 .
$$

In view of the normalization $v_{i \Omega^{\prime}}\left(\zeta_{0}\right)-v_{i \Omega}\left(\zeta_{0}\right)=0$ we conclude $[1, \mathrm{p} .147]$ on the asserted convergence.

For later reference (No. 10) we let $\Omega^{\prime} \rightarrow S$ while keeping $\Omega$ fixed in (8), and have 


$$
B_{\Omega}\left(v_{1}\right)+c_{1}=c_{1 \Omega}+D_{\Omega}\left(v_{1}-v_{1 \Omega}\right)
$$

For $\Omega \rightarrow S$ this gives

$$
\lim _{\Omega \rightarrow S} B_{\Omega}\left(v_{1}\right)=0
$$

7. While both $v_{0}$ and $v_{1}$ were needed in the convergence proof we shall only make use of $v_{1}$ in the sequel.

LEMMA 3. The function $t$ constructed in No. 4 and the limiting function $v_{1}$ in (6) are identical.

Proof. By definition, $t=L_{1} t$, and by virtue of the uniqueness of principal functions it suffices to show that $v_{1}=L_{1} v_{1}$. Let

$$
W_{1}=\left(D_{a}-a\right) \cup\left(D_{0}-\zeta_{0}\right) \cup D_{\beta}
$$

and let $\Omega$ contain $S-W_{1}$. Denote by $L_{1 \Omega}$ the $L_{1}$-operator acting on functions on $\alpha_{1}=\partial W_{1}$ and providing us with harmonic functions on $\bar{\Omega} \cap W_{1}$. Then $v_{1 \Omega}=L_{1 \Omega} v_{1 \Omega}$ and we are to prove that $\lim L_{1 \Omega} v_{1 \Omega}=L_{1} v_{1}$. On $\alpha_{1}$ we have $v_{1 \Omega} \rightarrow v_{1}$, and consequently

$$
L_{1 \Omega} v_{1 \Omega}-L_{1 \Omega} v_{1}=L_{1 \Omega}\left(v_{1 \Omega}-v_{1}\right) \rightarrow 0,
$$

that is, $\lim L_{1 \Omega} v_{1 \Omega}=\lim L_{1 \Omega} v_{1}$. By definition of $L_{1}$ [1], the latter expression is indeed $L_{1} v_{1}$.

The above reasoning for $t$ can also be applied to show that

$$
t_{0}=\lim _{\Omega \rightarrow S} t_{0 \Omega}
$$

where $t_{0 \Omega}$ is the $t_{0}$-function constructed on $\Omega$.

8. It is now easy to see that $s=s_{0}+t$ is symmetric:

LEMMA 4. For any $a, b \neq \zeta_{0}$,

$$
s(a, b)=s(b, a) .
$$

Proof. Again first suppose $S$ is a bordered compact surface with border $\beta$. Let $\alpha_{a}, \alpha_{b}, \alpha_{0}$ be peripheries of parametric disks about $a, b, \zeta_{0}$ and set $t_{a}=t(\zeta, a), t_{b}=t(\zeta, b)$ with $t_{a} \mid \beta=$ const., $t_{b} \mid \beta=$ const. Then

$$
\int_{\beta-\alpha_{0}-\alpha_{n}-\alpha_{b}} t_{a} d t_{b}^{*}-t_{b} d t_{a}^{*}=0 .
$$

Here $\int_{\beta}=0$, and, in the same manner as in No. 5, we obtain $\int_{\alpha_{0}}=4 \pi\left(s_{0}(a)-s_{0}(b)\right)$, Analogous computations give $\int_{\alpha_{a}}=4 \pi t_{b}(a)$ and $\int_{\alpha_{b}}=-4 \pi t_{a}(b)$. We infer that 


$$
s_{0}(a)+t(a, b)=s_{0}(b)+t(b, a) .
$$

This is (13).

If $S$ is noncompact the statement follows from the above and the uniform convergence of the approximating functions formed on the $\Omega \subset S$.

\section{§3. Conformal Metric.}

9. In terms of $s$ we introduce the conformal metric with area element $d \omega=\lambda^{2} d S$, where

$$
\lambda^{2}=\Delta s=\Delta s_{0}=\frac{e^{t_{0}}\left|\operatorname{grad} t_{0}\right|^{2}}{\left(1+e^{t_{0}}\right)^{2}} .
$$

For $x \in(-\infty, \infty)$ denote by $\alpha\left(x, t_{0}\right)$ the level line $t_{0}=x$ on $S-\zeta_{0}-\zeta_{1}$. When $x$ is near $-\infty$ or $\infty$, then $\alpha\left(x, t_{0}\right)$ is compact encircling $\zeta_{1}$ or $\zeta_{0}$, respectively. On a noncompact $S, \alpha$ is noncompact for some values $x$.

Given a regular region $\Omega \subset S$ containing $\zeta_{0}, \zeta_{1}$ set $\alpha_{\Omega}\left(x, t_{0}\right)=\alpha\left(x, t_{0}\right) \cap \Omega$.

LeMma 5. On a noncompact $S$, we have for $x \in(-\infty, \infty)$,

$$
\lim _{\Omega \rightarrow S} \int_{\alpha \Omega\left(x, t_{0}\right)} d t_{0}^{*} \leqq 4 \pi \text { a.e. }
$$

Proof. If the statement is false, then there exists a constant $\varepsilon>0$, a regular region $\Omega_{0}$, and a value $x_{0}$ with $\alpha_{0}=\alpha_{\Omega_{0}}\left(x_{0}, t_{0}\right)$, such that $\int_{\alpha_{0}} d t_{0}^{*}>4 \pi+3 \varepsilon$, $\operatorname{grad} t_{0} \mid \alpha_{0} \neq 0$, and $\alpha_{0}$ is not tangent to $\partial \Omega_{0}$. Moreover, there is a $\delta>0$ with $\operatorname{grad} t_{0} \mid G \neq 0$ and $\int_{\gamma}\left|d t_{0}^{*}\right|=\langle\varepsilon\rangle$, where $G=\Omega_{0} \cap\left\{\zeta|| t_{0}(\zeta)-x_{0} \mid<\delta\right\}, \gamma=\bar{G} \cap \bar{\Omega}_{0}$ consists of disjoint closed arcs $\gamma_{1}, \gamma_{2}$ joined by $\alpha_{0}$, and $\langle\varepsilon\rangle$ stands for a quantity in the interval $(-\varepsilon, \varepsilon)$.

Because of the uniform convergence $t_{0 \Omega} \rightarrow t_{0}$ there exists a regular region $\Omega \supset \Omega_{0}$ such that

$$
\begin{aligned}
\int_{\alpha_{0}} d t_{0 \Omega}^{*} & =\int_{\alpha_{0}} d t_{0}^{*}+\langle\varepsilon\rangle, \\
\int_{\nu}\left|d t_{0 \Omega}^{*}\right| & =\int_{\nu}\left|d t_{0}^{*}\right|+\langle\varepsilon\rangle=\langle 2 \varepsilon\rangle, \\
\left|t_{0 \Omega}-t_{0}\right| \mid G & <\delta .
\end{aligned}
$$

For any arc $\alpha \subset G$ from $\gamma_{1}$ to $\gamma_{2}$, we have

$$
\int_{\alpha} d t_{0 \Omega}^{*}=\int_{\alpha_{0}} d t_{0 \Omega}^{*}+\langle 2 \varepsilon\rangle=\int_{\alpha_{0}} d t_{0}^{*}+\langle 3 \varepsilon\rangle>4 \pi
$$

On the other hand, the arc $\alpha=\alpha_{\Omega_{0}}\left(x_{0}, t_{0 \Omega}\right)$ gives 


$$
\int_{\alpha} d t_{0 \Omega}^{*} \leqq \int_{\alpha_{\Omega}\left(x_{0}, t 0 \Omega\right)} d t_{0 \Omega}^{*}=4 \pi .
$$

This contradiction proves Lemma 5 .

10. We are now ready to show:

LEMмa 6. The total area of $S$ is

$$
\int_{S} d \omega=4 \pi \text {. }
$$

Proof. Let $\alpha_{1}: t_{0}=x_{1}$ and $\alpha_{2}: t_{0}=x_{2}$ be level lines such that the sets $\zeta_{1} \cup\left\{\zeta \mid t_{0}(\zeta) \leqq x_{1}\right\}$ and $\zeta_{0} \cup\left\{\zeta \mid t_{0}(\zeta) \geqq x_{2}\right\}$ are compact. We know from (11) that $\int_{\partial \Omega} t_{0} d t_{0}^{*} \rightarrow 0$ as $\Omega \rightarrow S$. For this reason the Dirichlet integral of $t_{0}$ over the region with $x_{1}<t_{0}<x_{2}$ is

$$
\begin{aligned}
D\left(t_{0}\right) & =\lim _{\Omega \rightarrow S} \int_{\alpha_{2}-\alpha_{1}+\partial \Omega} t_{0} d t_{0}^{*}=4 \pi\left(x_{2}-x_{1}\right) \\
& =\lim _{\Omega \rightarrow S} \int_{x_{1}}^{x_{2}} \int_{\alpha_{\Omega}\left(x, t_{0}\right)} d t_{0}^{*} d x .
\end{aligned}
$$

From this and from Lemma 5 we conclude that

$$
\lim _{\Omega \rightarrow S} \int_{\alpha_{\Omega}\left(x, t_{0}\right)} d t_{0}^{*}=4 \pi \text { a.e. }
$$

The lemma follows on integrating (14).

\section{§4. ANALYTIC MAPPINGS}

11. Let $R$ be an arbitrary noncompact Riemann surface and $R_{0}$ a parametric disk with border $\beta_{0}$. Take an adjacent regular region $\Omega$ with border $\beta_{0} \cup \beta_{\Omega}, \quad \bar{R}_{0} \cap \bar{\Omega}=\beta_{0}$, and a harmonic function $u$ on $\bar{\Omega}$ such that $u\left|\beta_{0}=0, u\right| \beta_{\Omega}=k$ (const.) with $\int_{\beta_{0}} d u^{*}=1$. Let $\beta_{h}$ be the level line $u^{-1}(h)$, $h \in[0, k]$ and denote by $\Omega_{h}$ the region $u^{-1}((0, h))$ with border $\beta_{0} \cup \beta_{h}$.

Suppose $\zeta=f(z)$ is a complex analytic mapping of $R$ into a compact or noncompact Riemann surface $S$. For $a \in S$ let $v(h, a)$ be the number of inverse images $z_{j}$ of $a$ in $\Omega_{h}$, counted with their multiplicities. To study value distribution under $f$ we use the counting function

the proximity function

$$
A(h, a)=4 \pi \int_{0}^{h} v(h, a) d h,
$$

$$
B(h, a)=\int_{\beta_{h}} s(f(z), a) d u^{*},
$$

the characteristic function 


$$
C(h)=\int_{\Omega_{h}}(h-u(z)) d \omega(f(z)),
$$

and an auxiliary function

$$
D(h, a)=B(0, a)+h B^{\prime}(0, a) .
$$

An application of Stokes' formula to the functions $s(f(z), a)$ and $h-u(z)$ over $\Omega_{h}$ less disks about $z_{j}$ shrinking to their centers gives the first main theorem [2]:

$$
A(k, a)+B(k, a)=C(k)+D(k, a) .
$$

12. Mappings that offer interest are those for which $k / C(k) \rightarrow 0$ as $\Omega \rightarrow S$. For these nondegenerate mappings, $D(k, a)=O(k)$ is negligible, and the $(A+B)$ affinity is the same for all points $a$. The defect of $a$ is

$$
\delta(a)=\liminf _{\Omega \rightarrow S} \frac{B(k, a)}{C(k)}=1-\limsup _{\Omega \rightarrow S} \frac{A(k, a)}{C(k)} .
$$

For a Picard point, $\delta(a)=1$, while for a "totally covered" point, $\delta(a)=0$. The main question is: Are the defect points $a$ with $\delta(a)>0$ "exceptional" in comparison with points with $\delta(a)=0$ ? No bounds independent of $f$ exist for the defect sum, for mappings with infinitely many Picard values are known. We shall show, however, that the set of defect points is small.

13. By way of preparation, we first prove:

LEMMA 7. Under an arbitrary $f$,

$$
\int_{S} B(k, a) d \omega(a)=\int_{S} D(k, a) d \omega(a) .
$$

Proof. We are at liberty to choose $R_{0}$ so that $f\left(\beta_{0}\right)$ does not meet $\zeta_{0} \cup \zeta_{1}$. The counting function can be written

$$
A(k, a)=4 \pi \sum_{z_{j} \in \Omega} v\left(z_{j}\right),
$$

where $v=k-u$. It follows that

$$
\begin{aligned}
\int_{S} A(k, a) d \omega(a) & =4 \pi \int_{S} \sum_{z_{j} \in \mathcal{S}^{-1}(a)} v\left(z_{j}\right) d \omega(a) \\
& =4 \pi \int_{\Omega} v(z) d \omega(f(z)) \\
& =4 \pi C(k)=\int_{S} C(k) d \omega(a) .
\end{aligned}
$$


On integrating the first main theorem with respect to $d \omega(a)$ over $S$ we obtain the lemma.

14. We decompose $\int D d \omega$ :

$$
\int_{S} B(k, a) d \omega(a)=\int_{S} B(0, a) d \omega(a)+k \int_{S} B^{\prime}(0, a) d \omega(a),
$$

and prove first:

LEMMA 8.

$$
\int_{S} B(0, a) d \omega(a)=O(1) .
$$

Proof. Cover $\gamma_{0}=f\left(\beta_{0}\right)$ by parametric disks $D_{i}, i=1, \cdots, N$, such that $\bigcup \bar{D}_{i}$ is disjoint from the parametric disks $D_{0}, D_{1}$ about $\zeta_{0}, \zeta_{1}$, and that slightly smaller disks $D_{i}^{\prime}$ concentric with $D_{i}$ already cover $\gamma_{0}$. Decompose $\gamma_{0}$ into closed arcs $\gamma_{i} \subset D_{i}^{\prime}, \bigcup \gamma_{i}=\gamma_{0}$.

We know that

$$
s(\zeta, a)=s(a, \zeta)=s_{0}(a)+t(a, \zeta) .
$$

For $\zeta \in \gamma_{i}$ the principal function $t(a, \zeta)$ is constructed from the singularities

$$
\sigma\left|D_{i}=2 \log \right| \frac{1-a \bar{\zeta}}{a-\zeta}|, \quad \sigma| D_{0}=2 \log \left|a-\zeta_{0}\right| .
$$

On $\partial D_{i}^{\prime}$ and on the periphery $\partial D_{0}^{\prime}$ of a disk $D_{0}^{\prime}$ slightly smaller than $D_{0}, \sigma=O(1)$. For this reason $t\left|D_{i}=\sigma+O(1), t\right| D_{0}=\sigma+O(1), t \mid S-D_{i}-D_{0}=O(1)$, and consequently $\int_{S} t(a, \zeta) d \omega(a)=O(1)$.

Similarly $t_{0}\left|D_{0}=-2 \log \right| a-\zeta_{0}\left|+O(1), t_{0}\right| D_{1}=2 \log \left|a-\zeta_{1}\right|+O(1)$, $t_{0} \mid S-D_{0}-D_{1}=O(1)$, and we inferfor $s_{0}=\log \left(1+e^{t_{0}}\right)$ that $\int_{S} s_{0}(a) d \omega(a)=O(1)$. In summary, $\int_{S} s(a, \zeta) d \omega(a)=O(1)$ and

$$
\int_{S} B(0, a) d \omega(a)=\int_{\gamma_{0}} \int_{S} s(a, \zeta) d \omega(a) d u^{*}=O(1) .
$$

15. We turn to the second term in the decomposition of $\int D d \omega$ :

LEMMA 9.

$$
\int_{S} B^{\prime}(0, a) d \omega(a)=O(1)
$$

Proof. Since $\zeta_{0} \notin \gamma_{0}$, we have $\int_{\gamma_{0}} d s_{0}^{*}=O(1)$. The integral $\int_{\gamma_{0}} d t^{*}$ gives the winding number of $\gamma_{0}$ about $\zeta_{0}$ and is finite. Consequently

$$
\int_{\gamma_{0}} d s^{*}=\int_{\gamma_{0}} d s_{0}^{*}+\int_{\gamma_{0}} d t^{*}=O(1)
$$

and $\int_{S} \int_{\gamma_{0}} d s^{*} d \omega=O(1)$. This proves the lemma. 
16. We have established the result we set out to find:

THEOREM. Under a complex analytic mapping of an arbitrary Riemann surface into another arbitrary Riemann surface the set of defect points is so small that

$$
\int_{S} B(k, a) d \omega(a)=O(k)
$$

In view of $A(k, a)+B(k, a)=C(k)+O(k)$ this poses a strong restriction on the $B(k, a)$ under nondegenerate mappings $C(k) / k \rightarrow \infty$. In contrast with the general affinity relation (see [2])

$$
\Sigma \alpha+\Sigma \beta+\Sigma \psi \leqq 2+\eta,
$$

which holds for nondegenerate functions satisfying an additional growth condition, (22) is valid for every nondegenerate mapping.

Added in proof. Lemma 6 has interest in its own right: it shows that in our metric an arbitrary Riemann surface behaves much like a sphere.

\section{BIBLIOGRAPHY}

1. L. Ahlfors and L. Sario, Riemann surfaces, Princeton Math. Series, No. 26, Princeton Univ. Press, Princeton, N. J., 1960.

2. L. Sario, General value distribution theory, Nagoya Math. J. 23 (1963), 213-229.

3. - An integral equation and a general existence theorem for harmonic functions, Comment. Math. Helv. 38 (1964), 284-292.

UNIVERSITY OF CALIFoRnia,

Los ANgeles, California 